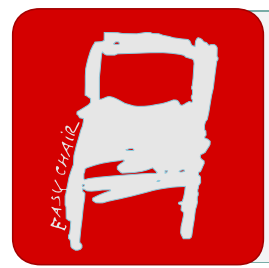

EPiC Series in Health Sciences

\title{
No difference in fifteen-year survival after navigation-assisted medial pivot or central pivot mobile bearing total knee arthroplasty.
}

\author{
Jean-Yves Jenny, Dominique Saragaglia, Thierry Gaillard, Frédéric Châtain \\ University Hospital Strasbourg, Strasbourg, France \\ jean-yves . jenny@chru-strasbourg.fr
}

\begin{abstract}
The survival rate of navigation-assisted mobile bearing total knee arthroplasty when mechanical revision was considered as the end-point wa snot impacted by the design, either central pivot or medial pivot.
\end{abstract}

\section{Introduction}

Total knee arthroplasty (TKA) is considered a highly successful procedure. Survival rates of more than $90 \%$ after 10 years are generally reported (Baumbach 2016). Computer assistance has been suggested to improve the accuracy of implantation of a TKA (Jenny 2005). Mobile bearing designs are supposed to decrease polyethylene wear (Jenny 2013). Furthermore, it has been postulated that mobile bearing TKAs with a more physiological medial pivoting axis (MPA) may offer a better outcome than conventional TKAs with a central pivoting axis (CPA) (Cacciola 2020). The present study was designed to evaluate the long-term (more than 10 years) results of TKAs which were routinely implanted with help of a non-image based navigation system and to compare the respective survival of MPA and CPA TKAs.

The hypothesis of this study was that the 10- to 15-year survival rate of MPA TKAs will be improved in comparison to CPA TKAs. 


\section{Material and methods}

A multicentric retrospective study has been implemented. 1,604 navigated TKAs were included: 545 males and 1,059 females with an average age of 70 years and an average body mass index (BMI) of $30 \mathrm{~kg} / \mathrm{m}^{2}$. 1.154 cases could be paired into CPA (control group-577 cases) and MPA (study group577 cases) after a logistic regression analysis of age, gender, body mass index and severity of the coronal deformation, defining the propensity score for each case.

All patients were contacted for the purpose of this study to determine the survival of TKA. The reason for a possible revision was noted. The main criterion was the occurrence of a revision for any cause. Survival curves were calculated, and the influence of pivoting axis location was analyzed by a logrank test at a 5\% level of significance. Oxford scores and Knee Society score were calculated, and the difference was analyzed with a Student test at a 5\% level of significance.

\section{Results}

There was no significant difference between control and study groups for any baseline data. 208 patients deceased prior to the study (18\%) and were included as censored data at the time of death. 276 cases were lost of follow-up (24\%) and were included as censored data at the time of last available clinical examination. Final follow-up was obtained for 670 cases (58\%).

22 prosthetic revisions were performed for mechanical reasons during the follow-up time (2\%). The global survival rate after 14 years was $97 \%$. There was no significant difference between the 13 year survival rates of CPA $(98 \%)$ and MPA $(97 \%)$ TKAs. There was no significant difference between both groups for final Oxford and Knee Society scores.

\section{Discussion}

The biomechanics of a normal knee is different between the medial femorotibial joint (more stable) and the lateral one (more mobile). It seems logical to adapt the design of a TKA to that feature (Warth2017). The medialization of the pivoting center of a mobile bearing TKA is supposed facilitating or even inducing a more physiologic kinematics (Meneghini 2017). However, the primary hypothesis of this study was not confirmed: no difference was observed between CPA and MPA TKAs when considering the 13-year survival rate for mechanical revision or clinical results. The results of the present study do not support the assumption that the medialization of the pivoting center of a mobile bearing TKA is supposed improving clinical results or survival 


\section{References}

1. Baumbach JA, Willburger R, Haaker R, Dittrich M, Kohler S, 10-year survival of navigated versus conventional TKAs: a retrospective study, Orthopedics, 39(3 Suppl), pp: S72-76, 2016.

2. Jenny JY, Clemens U, Kohler S, Kiefer H, Konermann W, Miehlke RK, Consistency of implantation of a total knee arthroplasty with a non-image-based navigation system: a case-control study of 235 cases compared with 235 conventionally implanted prostheses, J Arthroplasty, 20(7), pp: 832-839, 2005.

3. JennyJY, Miehlke R, Saragaglia D, Geyer R, Mercier N, Schoenahl JY, Thiel B, Single-radius, multidirectional total knee replacement, Knee Surg Sports Traumatol Arthrosc, 21, pp: 2764$2769,2013$.

4. Cacciola G, De Martino I, De Meo F, Does the medial pivot knee improve the clinical and radiographic outcome of total knee arthroplasty? A single centre study on two hundred and ninety seven patients, Int Orthop 44(2), pp::291-299, 2020.

5. Warth LC, Ishmael MK, Deckard ER, Ziemba-Davis M, Meneghini RM, Do medial pivot kinematics correlate with patient-reported outcomes after total knee arthroplasty? J Arthroplasty. 32(8), pp:2411-2416, 2017.

6. Meneghini RM, Deckard ER, Ishmael MK, Ziemba-Davis M, A dual-pivot pattern simulating native knee kinematics optimizes functional outcomes after total knee arthroplasty, J Arthroplasty, 32(10), pp:3009-2015, 2017..

\section{Disclosure}

Jean-Yves JENNY receives royalties from AESCULAP, is a paid consultant for FH Orthopedics and Globus Medical, is member of the board of the CAOS-International Society and of the International Society for Technology in Arthroplasty. Dominique SARAGAGLIA receives royalties from AESCULAP. Thierry GAILLARD receives royalties from AMPLITUDE. Frédéric CHATAIN receives royalties from AMPLITUDE and DEDIENNE. 